\title{
HACIA UN MODELO SOCIOCULTURAL EXPLICATIVO DEL ALTO RENDIMIENTO Y DE LA ALTA CAPACIDAD I: ÁMBITO FAMILIAR
}

\author{
TOWARDS A SOCIO-CULTURAL EXPLICATIVE MODEL OF HIGH \\ ACHIEVEMENT AND HIGH ABILITY I: FAMILY CONTEXT
}

\author{
C. Jiménez Fernández*, B. Álvarez González**, \\ J. A. Gil Pascual*** y $M^{a} A$. Murga Menoyo**** \\ de la U.N.E.D. \\ José A. Téllez Muñoz***** \\ de la Universidad de Cádiz \\ y $M^{a} P$. Trillo Miravalles******
}

\section{RESUMEN}

En este artículo proponemos un Modelo sociocultural explicativo del alto rendimiento y de la alta capacidad. El modelo es consecuencia, por un lado, de la propia evolución que la psicología, y cien-

* Carmen Jiménez Fernández (mjimenez@edu.uned.es) es catedrática en el Departamento MIDE I en la Ftad. De Educación de la UNED. Dirige la línea de investigación "Educación, alta capacidad y género" y el postgrado "Experto Universitario en Diagnóstico y educación de los alumnos con Alta Capacidad"

** Beatriz Alvarez González (balvarez@edu.uned.es), Profesora Titular de Orientación Familiar en el Dpto. MIDE II de la Ftad. de Educación de la UNED. Su trayectoria investigadora incluye temas de orientación familiar, alta capacidad y género, y educación intercultural.

*** Juan Antonio Gil Pascual (jgil@edu.uned.es), Profesor Titular de Métodos de Investigación en Educación en el Dpto. MIDE I de la UNED. Matemático y Drto. en Educación. Con dilatada docencia en todos los niveles educativos y como experto en estudios de opinión. Participante en diversos proyectos de investigación subvencionados como analista de datos. Premio Nacional de Investigación Educativa 1998.

**** $M^{a}$ Ángeles Murga Menoyo (mmurga@edu.uned.es), es Profesora Titular y actualmente Directora del Departamento de Teoría de la Educación y Pedagogía Social de la UNED. Su trayectoria investigadora incluye temas de innovación educativa, enseñanza a distancia y educación en valores.

***** José A. Téllez.Muñoz (jose.tellez@uca.es), es Profesor Asociado de la Universidad de Cádiz, Departamento de Didáctica (Área MIDE) y miembro del Centro INTER de Investigación en Educación Intercultural (www.uned.es/centrointer). Su trayectoria investigadora incluye temas de: comprensión lectora, alta capacidad y educación intercultural.

****** M $M^{a}$ Paz Trillo Miravalles (mptrillo@bec.uned.es), Licenciada en Psicopedagogía y doctoranda en la UNED. Actualmente becaria en la Cátedra UNESCO de Educación a Distancia (www.uned.es/cued). Sus líneas de investigación se centran en: educación, capacidad y género, y educación vial. 
cias afines, como la pedagogía y la orientación educativa, han sufrido a lo largo de las últimas décadas, tendiéndose cada vez más a integrar variables de tipo cognitivas, afectivas y socioculturales; y por otro, de la propia evolución y desarrollo de las investigaciones realizadas por el equipo, especialmente de la información emergente y no prevista surgida durante el proceso de investigación. Contar con un modelo amplio y comprensivo resulta esencial para garantizar los procesos de orientación e intervención psicopedagógica que favorezcan un alto desarrollo y rendimiento y una atención adecuada a los estudiantes con altas capacidades. En el modelo se concretan seis dimensiones, en este artículo nos centraremos en una de ellas: el ámbito familiar.

Palabras clave: Alta capacidad, alto rendimiento, género, psicología cultural, familia.

\begin{abstract}
In this article we propose a Sociocultural explanatory model of high perfomance and high ability. This model is the result of, o one hand, the evolution of Psychology and related science, like pedagogy and education al guidance, during the last decades, towards the integration of cognitive, emotional and sociocultural variables, and, on the other hand, the result of the evolution and development of the research carried out by our own group, especially regarding the unexpected information come up during the research process. To have a wide and understanding model is essential to guarantee the psychopedagogical intervention and guidance processes which favour a high development and performance, as well as, an appropriate attention to the students with high abilities. This model includes six aspects, in the present article we focus on one of them: the family field.
\end{abstract}

Key words: High ability, high performance, gender, cultural psychology, family.

\title{
Introducción
}

Cualquier proceso de investigación se apoya en unas bases conceptuales y teóricas. En nuestro caso, han sido las aportaciones de la psicología, en interacción con otras disciplinas, durante estos últimos 60 años, las que nos han ayudado a estar atentos a diferentes variables que considerábamos esenciales para comprender qué factores condicionan el alto rendimiento y la alta capacidad.

En este artículo pretendemos esbozar un Modelo sociocultural explicativo del alto rendimiento y de la alta capacidad. Contar con un modelo amplío y comprensivo de referencia resulta esencial para desarrollar una intervención orientadora y educativa que favorezca el alto rendimiento y una adecuada atención a los estudiantes con alta capacidad. La configuración de este modelo de referencia que proponemos integra tanto las aportaciones teóricas que sobre el tema existen como la información emergente de las investigaciones realizadas por el grupo.

Comenzaremos señalando las aportaciones teóricas que sobre el tema existen, prestando especial atención a los cambios de enfoque que la investigación en psicología ha sufrido durante las últimas décadas. Por otro lado, reflexionaremos sobre la necesidad de ser receptivos a la información implícita que se genera durante el proceso de investigación. La confluencia de estos dos aspectos es lo que nos ha llevado a comenzar a definir el modelo de referencia que proponemos.

Posteriormente, detallaremos cuáles han sido los objetivos de nuestra investigación, la muestra de estudio, y cuáles han sido los procedimientos de recogida de información utilizados, concretando a su vez las variables de estudio. Una vez clarificado el diseño de investigación, y atendiendo a la flexibilidad metodológica que reclamamos, pasamos a detallar los resul- 
tados alcanzados, centrándonos especialmente en la información de tipo cualitativa, complementándola a su vez con información procedente de los cuestionarios aplicados. Terminamos señalando las conclusiones del estudio y realizando una breve valoración crítica del proceso de investigación con la intención de que nos permita orientar investigaciones posteriores.

\section{Marco conceptual: desde la psicología cognitiva hacia una psicología cultural y emocional ${ }^{1}$}

La psicología cognitiva ha tenido la virtud de ayudarnos a clarificar en gran medida cómo funciona la mente humana. Pero lamentablemente en muchas ocasiones las explicaciones dadas han resultado ser excesivamente mecanicistas, desvirtuándose en gran medida su sujeto de estudio (Bruner, 1990, 2006; Téllez, 2005).

Al amparo de la ciencia cognitiva surgieron modelos explicativos de la alta capacidad. Se equiparaba la alta capacidad a inteligencia, y frente a modelos generales (Sperman, 1927) y factoriales (Thurstone, 1938; Guilford, 1967) cobraron una mayor importancia modelos que se centraban sobre todo en los procesos cognitivos y metacognitivos puestos en funcionamiento (Sternberg, 1991; aunque atiende también a variables contextuales). Más allá de estas variables es necesario considerar factores afectivos, emocionales y motivacionales. Desde líneas de investigación probablemente paralelas en psicología se han definido innumerables modelos definitorios de estos dos bloques de variables, las cognitivas y las afectivas, no siendo prácticamente hasta la década de los noventa cuando se han intentado integrar de alguna manera (Renzulli, 1994).

La concepción de la inteligencia sigue ampliándose en un intento de responder a las variadas capacidades humanas. En esta línea, el mejor ejemplo que tenemos son los modelos de inteligencias múltiples propuestos por Howard Gardner $(1983,1999)$. En nuestro contexto cabe destacar las aportaciones de Prieto y col. $(2002,2004)$ que desde la Universidad de Murcia están utilizando este modelo para el diagnóstico de alumnos con alta capacidad.

Por otro lado, cabe recordar que es a partir principalmente de la recuperación de la psicología sociohistórica y cultural cuando se ha comenzado a considerar a la mente humana en contexto, intentando ir más allá de explicaciones con pretensiones universales y apoyadas principalmente en estudios de laboratorio. A partir de la década de los ochenta bajo los rótulos de psicología cultural, psicología transcultural o etnopsicología se comienzan a acoger estrategias metodológicas y de investigación propias de otras disciplinas como la antropología². Autores, y sólo por citar algunos, como Michael Cole, James Werstch, Jerome Bruner, o Barbara Rogoff, son claros representantes de este enfoque de estudio. La International Society for Cutural and Activity Research (ISCAR) ${ }^{3}$ es uno de los organismos pro-

1. Una breve descripción de las teorías y modelos explicativos de la alta capacidad que señalamos a continuación la puede encontrar en Jiménez (2000, 2002).

2. Esta intencionalidad interdisciplinar estaba en los orígenes de la psicología cognitiva. Pero de nuevo las influencias e interacciones más intensas se producen entre disciplinas como la psicología y las ciencias de la computación, sobre todo debido a la proliferación del enfoque del procesamiento de la información o enfoques posteriores como el conexionismo. Las interacciones habidas con la antropología resultan menores, si no nulas. A pesar de ello compartimos con Jerome Bruner (2006) que la separación entre disciplinas como la psicología y la antropología resulta arbitraria.

3. La web de la ISCAR es: http://www.iscar.org. 
motores de esta necesidad contextualizadora. Las construcciones de significados, individuales y compartidas, las actividades humanas y las interacciones sociales comienzan a ser objeto de estudio. De manera cercana a las exigencias de este enfoque y desde el estudio de la alta capacidad cabe destacar la adaptación que del modelo de Renzulli realiza Mönks (Mönks y van Boxtel, 1988; Mönks y Mason, 2000) y desde una perspectiva psicosocial las aportaciones de Tannebaum (1991) o el modelo de Gagné (1991).

\section{La ciencia escondida: flexibilidad del proceso de investigación}

Los procesos de investigación son en muchas ocasiones mucho más abiertos e intuitivos de lo que con normalidad se suele mostrar. Con mucha frecuencia en las publicaciones científicas se intenta mostrar un proceso de investigación cerrado, con la intención de ajustarnos a la rigurosidad y sistematicidad que desde la ciencia se nos pide. Desvirtuamos el proceso ocultándose en muchos casos lo que sucede en la trastienda.

Estamos convencidos de que durante el proceso de investigación, a pesar de intentar ajustarnos al protocolo de actuación que el método científico nos ofrece, y en muchos casos nos impone, debemos estar abiertos a la nueva información que va surgiendo, a las nuevas interpretaciones que vamos realizando (que en ocasiones no tienen porque responder a ninguna hipótesis u objetivo planteado previamente), a nuevas pistas que nos ayuden a reconducir la propia investigación y a centrar nuestra atención en los aspectos más constitutivos de nuestro ámbito de estudio.

Los constructos teóricos que asumimos al comienzo de la investigación deben ir dibujándose, nuestros objetivos pueden ampliarse e incluso ayudarnos a cambiar el enfoque de estudio, las aproximaciones metodológicas se van afinando. Frente a la sistematicidad y rigurosidad de la ciencia es necesario reclamar procesos más flexibles que permitan ajustarnos a los aspectos que estudiamos. Es necesario hacer visibles aquello que normalmente no se suele contar, debemos dejar ver cómo se hace la ciencia.

Durante esta investigación, como veremos más adelante, nos hemos visto influidos por el propio proceso de la misma, llevándonos a centrar nuestra atención en nuevas variables de estudio que inicialmente el equipo de investigación no tenía previstas. Es a partir de ahí cuando nuestra intencionalidad se amplia y cuando nos proponemos como objetivo la definición de un Modelo sociocultural explicativo del alto rendimiento y de la alta capacidad.

\section{Método}

El objetivo general ${ }^{4}$ de nuestro estudio era inicialmente intentar: "definir de la manera más clara posible cuáles eran las características personales y las condiciones familiares, escolares y sociales que hace posible el alto rendimiento".

Para alcanzar dicho objetivo nos centramos en una muestra caracterizada de por sí por un alto rendimiento, han sido los alumnos y alumnas con Beca de Excelencia (BE) de la Comunidad de Madrid. Para poder optar a las BE en el inicio de los estudios universitarios

4. Remitimos a los interesados al informe de la investigación donde se detallan el conjunto de objetivos planteados, los instrumentos y procedimientos de recogida de la información, resultados y conclusiones. 
es requisito haber obtenido una media de sobresaliente a lo largo de los estudios de bachillerato. Con una muestra así conseguimos mantener un perfil similar al de estudios previos realizados por el grupo de investigación (Jiménez y col. 2001, 2002a) donde nuestra atención se focalizó en los alumnos y alumnas que obtenían Premio Extraordinario de Bachillerato (PEB) permitiéndonos en cierta medida establecer algunas comparaciones, ya que muchos de los estudiantes que obtienen BE han sido alumnos y alumnas con PEB, en nuestro caso el $36 \%$. La muestra participante ha estado conformada por 40 chicos y 78 chicas, un total de 118 estudiantes de los cuales la mayoría cursan su primer año de la carrera universitaria.

Para alcanzar el objetivo general propuesto integramos métodos de recogida de información tanto cuantitativos como cualitativos (en este artículo nos centramos principalmente en la información recogida a través de la técnica cualitativa que señalamos a continuación).

Como técnicas cuantitativas se aplican los siguientes tests y cuestionarios (ver Tabla 1, los cuatro últimos cuestionarios señalados han sido elaborados por miembros del equipo de investigación).

Como estrategia cualitativa de recogida de la información se ha utilizado el grupo de discusión. Hemos tenido 8 sesiones con una participación total de 91 estudiantes 59 chicas y 32 chicos). Los grupos estaban orientados a extraer información tanto de las variables personales como contextuales. Después del análisis de la información transcrita ${ }^{5}$ organizamos la información en 6 grandes dimensiones (ámbito familiar, ámbito académico, capacidades personales, amistades y relaciones de pareja, ámbito laboral y ámbito social) que nos sirven por un lado, para complementar los datos obtenidos a través de los distintos cuestionarios y tests utilizados, y por otro para, explorar nuevas variables que al comienzo de la investigación no estaban en nuestro foco de estudio.

Este intento de atención a las percepciones, opiniones, valoraciones, que los participantes en la investigación, alumnos y alumnas con Beca de Excelencia en la Universidad, han mostrado durante los grupos de discusión, nos han llevado a plantearnos, aunque en muchos casos, tan solo de manera exploratoria, una diversidad de aspectos que nos ayudan a conformar una visión amplia de la situaciones personales que éstos vienen viviendo, cómo las perciben, cuáles son las influencias que han recibido, qué papel juegan en la sociedad, qué expectativas encuentran, qué cambios sociales se están dando y qué cambios se están reclamando. El modelo explicativo que estamos empezando a esbozar se centra en las seis dimensiones que hemos apuntado anteriormente. En la figura 1 se señalan las posibles interacciones y los diferentes elementos analizados durante el proceso de análisis de la información recogida a través de los grupos de discusión.

\section{Resultados}

Para detallar los resultados seguiremos la estructuración definitiva alcanzada a partir del análisis de la información recogida a través de los grupos de discusión, centrándonos, por

5. Todas las sesiones fueron grabadas en audio. Para organizar la información en las diferentes categorías extraídas nos hemos apoyado en el programa de análisis cualitativo MaxQDA-2. 
TABLA 1: Pruebas aplicadas y variables de estudio.

\begin{tabular}{|c|c|c|}
\hline \multirow{2}{*}{ Prueba aplicada } & \multicolumn{2}{|c|}{ Variables de estudio } \\
\hline & Personales & Contextuales \\
\hline $\begin{array}{l}\text { PMA (Aptitudes Mentales Prima- } \\
\text { rias) (Thurstone y col. 1999) }\end{array}$ & $\begin{array}{l}\text { - Comprensión verbal } \\
\text { - Concepción espacial } \\
\text { - Razonamiento } \\
\text { - Cálculo numérico } \\
\text { - Fluidez verbal }\end{array}$ & \\
\hline $\begin{array}{l}\text { Cuestionario de procesos, orien- } \\
\text { taciones y concepciones del } \\
\text { aprendizaje y enseñanza del } \\
\text { alumnado universitario (adapta- } \\
\text { ción de Marín, } 2000 \text { del Inventory } \\
\text { of Learning Styles in Higher Edu- } \\
\text { cation, de Vermunt) }\end{array}$ & $\begin{array}{l}\text { - Estrategias de estudio } \\
\text { - Motivaciones para el estudio } \\
\text { - Percepciones sobre el estuido }\end{array}$ & \\
\hline $\begin{array}{l}\text { Matrices progresivas (Raven, } \\
\text { 1992) }\end{array}$ & $\begin{array}{l}\text { - Manejar constructos no verbales } \\
\text { - Deducción de relaciones } \\
\text { - Percepción global para la solu- } \\
\text { ción del problema }\end{array}$ & \\
\hline $\begin{array}{l}\text { Cuestionario de valores persona- } \\
\text { les SPV (Gordon, 1999) }\end{array}$ & - Valores personales & \\
\hline $\begin{array}{l}\text { Cuestionario sobre pensamiento } \\
\text { matemático (Gil, 2003) }\end{array}$ & $\begin{array}{l}\text { - Estrategias cognitivas y emocio- } \\
\text { nales implicadas en la resolu- } \\
\text { ción de problemas matemáticos }\end{array}$ & \\
\hline $\begin{array}{l}\text { Cuestionario de sostenibilidad } \\
\text { (Murga, 2003) }\end{array}$ & $\begin{array}{l}\text { - Percepciones, actitudes y valo- } \\
\text { res acerca de la sostenibilidad }\end{array}$ & \\
\hline $\begin{array}{l}\text { Cuestionario de género (Jiménez } \\
\text { y Álvarez, 2003) }\end{array}$ & $\begin{array}{l}\text { - Rasgos personales } \\
\text { - Expectativas personales, educa- } \\
\text { tivas y profesionales }\end{array}$ & $\begin{array}{l}\text { - Entorno y clima familiar } \\
\text { - Ámbito de la escuela y } \\
\text { de la universidad } \\
\text { - Mis amigas y mis ami- } \\
\text { gos }\end{array}$ \\
\hline $\begin{array}{l}\text { Cuestionario evolutivo general } \\
\text { (Jiménez y Álvarez, 2003) }\end{array}$ & & $\begin{array}{l}\text { - Ámbito familiar, escolar } \\
\text { y extraescolar }\end{array}$ \\
\hline
\end{tabular}

motivos de espacio, exclusivamente en una de las seis dimensiones analizadas: ámbito familiar (el resto de dimensiones se desarrollan en otros artículos actualmente en preparación). Dada la amplitud del informe en cada una de las dimensiones nos centraremos exclusivamente en algunos de los focos, a nuestro juicio, más constitutivos del alto rendimiento, ilustrándolas con extractos literales de las transcripciones de los grupos y contrastándolas con la información procedente de los cuestionarios aplicados. Por otro lado, también reseñaremos brevemente información destacada por los chicos y chicas participantes que probablemente va más orientada a las percepciones y valoraciones que hacen de diferentes situaciones y condicionantes sociales. 


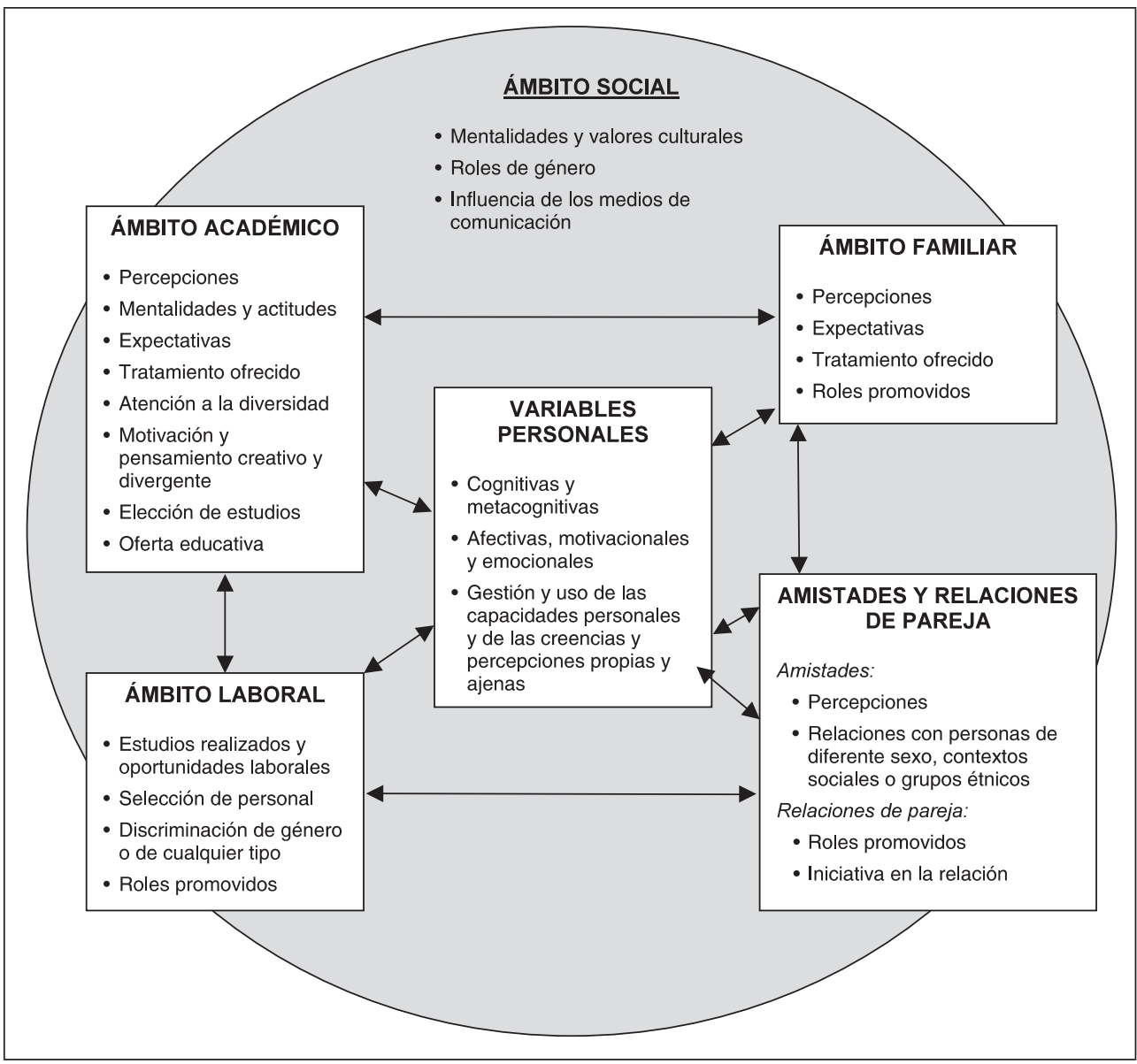

FIGURA 1.

Modelo explicativo del alto rendimiento y de la alta capacidad.

\section{Ámbito familiar}

La familia es uno de los núcleos sociales más influyentes en el desarrollo del individuo, en su forma de percibir la realidad y en la forma de enfrentarse a la misma. En trabajos previos del grupo (Jiménez y col. 2001, 2002a) se ha evidenciado que el papel jugado por las familias era una de las principales variables explicativas del alto rendimiento, por encima del papel de la escuela. Otra de las conclusiones de estos estudios era que los chicos y chicas con PEB proceden de familias de clase social media y media-alta. Los estudiantes de clases sociales bajas tenían vetado el camino hacia los Premios Extraordinarios de Bachillerato. 
A continuación señalaremos cuáles son los patrones que caracterizan a las familias de los chicos y chicas con altas capacidades que han participado en esta investigación ${ }^{6}$. Nos centraremos principalmente en las expectativas y roles que se promueven en las familias. También describiremos brevemente dos bloques de contenido que surgieron en las discusiones: la hora de llegada a casa y valoraciones en torno a la violencia de género.

\section{Expectativas de las familias hacia los chicos y chicas}

Los padres y madres de los entrevistados parecen tener iguales expectativas de desarrollo hacia chicos y chicas, intentando favorecer que todos sus hijos e hijas tengan unas mismas oportunidades formativas:

"Chico: En mi caso personal, por ejemplo yo tengo tres hermanas mayores y tres hermanos menores, $y$ entonces pues yo trato diferenciado a priori no he notado ninguno, eso si mis padres siempre se han preocupado de que más o menos fuésemos bien en los estudios pero no a nivel de exigirnos tal o cual sino a más preocupación. Yo creo que diferencia en mi caso ninguna" (Grupo 3).

"Chica: En mi casa somos dos. Mi hermano tiene 15 años, es tres años menor que yo, y en ningún caso ha habido discriminación, ni en la educación, ni en otro aspecto" (Grupo 4).

Los chicos y chicas consideran que influirán de manera positiva a sus hermanos o hermanas en el desarrollo de sus capacidades:

"Chico: Los padres siempre estarían ahí, pero yo le animaría para que hiciera lo mío a una hermana" (Grupo 5).

Los padres muestran patrones adaptativos evitando establecer comparaciones entre hermanos:

"Chica: En mi casa es al contrario. Yo sólo tengo una hermana y hemos ido al mismo instituto y los profesores cuando mi hermana ha tenido algún bache, decian que era porque mis padres me ponían a mí como ejemplo y eso es totalmente al contrario. Nunca me han dado a mi más importancia porque tuviera notas más brillantes, siempre a mi me han quitado importancia para que mi hermana no se sintiera mal y de hecho ahora está siguiendo los mismos pasos que yo, pero somos como la noche y el día. Aunque nos eduquen igual hay que tratar esas diferencias... En mi casa soy la mayor de tres hermanas y mi hermana pequeña le cuesta un poco más estudiar, (es que es un poco vaga), (risas), entonces a nosotros, aunque yo siempre he sacado mejores notas, nunca le han quitado mérito, ha sido siempre igual, se trata de que saques buenas notas, sin tener en cuenta las capacidades, o lo que sea" (Grupo 3).

Los padres y madres varían las expectativas en función de las capacidades e intereses de los chicos, no viéndose condicionadas por el sexo. Sí parecen influir los propios intereses de los padres a la hora de orientar hacia estudios o aficiones:

6. En Álvarez (2004) podemos encontrar una revisión sobre los factores del entorno familiar que condicionan el desarrollo de los chicos y chicas con alta capacidad. 
"Chica: En mi casa mi hermana también es pequeña, tiene 12 años y lo que si que se ha intentado es potenciar lo que cada una hace mejor. Por ejemplo: ella es mucho más creativa y va a dibujo a música y yo sin embargo me apuntaron a inglés desde muy pequeña, mi hermana dice que "pasa" del inglés y quiere apuntarse a italiano aunque no le sirva para nada, bueno para nada no (risas), que no es tan normal" (Grupo 3).

"Chica: Mi madre es profesora y tiene pedagogía y mi padre es ingeniero es como justo los dos roles. Mi padre intentaba que me gustara la física y las matemáticas y mi madre lo suyo y si hubiera tenido un hermano pues igual" (Grupo 4).

Los padres, principalmente la madre, favorecen una mayor independencia en las chicas a nivel formativo con la intención de favorecer una mayor independencia económica. Este nivel de independencia también se promueve en los chicos, promoviendo mayor autonomía con respecto a las tareas del hogar:

"Chica: Mi madre igual. Mi padre trabaja y mi madre no, y me dice que sea independiente económicamente" (Grupo 3).

Algunos padres muestran expectativas relativas a la elección de estudios asociadas al prestigio social y económico de las diferentes carreras. En otros casos los padres respetan los intereses y gustos de los chicos, independientemente de clichés sociales.

\section{Roles promovidos, asumidos o cuestionados}

"Chica: De cara a la galería se está intentando hacer esto pero tú coges un libro de primaria y pone: enfermera, y ves una chica o te pone: ingeniero, y ves un chico, entonces la educación no es la educación a partir de la universidad o desde el bachillerato, sino desde el principio y claro ves a las niñas que parecen repollos con lazo y los chicos que todos juegan al fútbol" (Grupo 2).

Los chicos perciben que ciertos juegos y juguetes se asocian a chicos o chicas. En ocasiones los compañeros de juegos aceptan que chicos o chicas jueguen a determinados juegos considerados como propios del sexo contrario. En otras ocasiones, los compañeros realizan críticas, principalmente a las chicas, por jugar a deportes o juegos "típicamente" masculinos (fútbol, baloncesto, etc.). En ocasiones los padres critican los comportamientos de los hijos en cuanto a la elección de juegos.

"Chica: Yo creo que en la sociedad lo de los juguetes no está superado (risas) y si lo piensas es grave. Mi experiencia es la contraria a la tuya, yo jugaba al fútbol y mis compañeros del colegio lo aceptaban pero en mi casa no. Si me pedía un patinete en reyes lo veían raro. Yo creo que ahora mismo no está superado" (Grupo 3).

"Chica: Cuando yo jugaba al baloncesto te das cuenta que tú eres la única chica y aunque estés sola no te pasan a ti, prefieren pasarla a uno que tiene a cuatro" (Grupo 7).

En lo relativo a los chicos se cuestiona la posibilidad de una construcción de una identidad masculina: 
"Chica: Eso depende, es como decía yo, que a los chicos se les está inculcando siempre: machismo, machismo; y ven una muñeca y normalmente no van a jugar a no ser que le hayan educado más liberal" (Grupo 4).

"Chico: No sé da cuenta, por eso prefiere jugar con una muñeca. Pero el padre no lo prefiere porque le van a decir que su hijo es "marica". Y eso ocurre, ha ocurrido siempre. Lo dicen de "cachondeo", pero lo piensa y esa es la pena" (Grupo 4).

En otras ocasiones encontramos actitudes y comportamientos de aceptación por parte de los padres:

"Chica: Yo por ejemplo de pequeña mi padre me enseñó a jugar al fútbol y a mí me acabó gustando el fútbol" (Grupo 2).

Con respecto a las tareas del hogar, se promueve que las chicas se responsabilicen con mayor frecuencia que los chicos, aunque existen excepciones en ambos sentidos (mayor participación de los chicos, y poca o nula participación de las chicas). En cualquier caso, gran parte de la responsabilidad sigue recayendo en las madres, también existen excepciones en los que la mayor carga la llevan los padres. En algunos casos se da un equilibrio en la repartición de tareas entre todos los miembros de la familia. En ocasiones, se acepta ideológicamente una mayor igualdad pero en la práctica se siguen manteniendo una mayor participación por parte de las chicas. Los chicos consideran que el modelo de los padres es un buen camino para favorecer una distribución equitativa de las tareas del hogar:

"Chica: Sí, pero yo creo que lo que están tratando de trasmitirnos nuestros padres es que no pase lo que pasa ahora, porque ellos están en esa fase de que la mujer hace las tareas de la casa y ellos quieren que no nos ocupemos nosotros solos de la casa. Entonces a lo mejor en la siguiente generación no pasa lo que está pasando con nuestros padres sino que nosotros consigamos cambiarlo para que nos preocupemos por igual de las tareas. Pero todavía no se ha conseguido" (Grupo 3).

Los chicos y chicas perciben que las madres renuncian con mayor frecuencia a la vida laboral para asumir la educación y cuidado de los hijos. Los chicos valoran de manera negativa estas renuncias, piensan que debería favorecerse un igual desarrollo profesional y una colaboración compartida de las tareas. Se solicitan medidas legislativas que favorezcan la conciliación de la vida familiar y laboral. En los casos, que se debiera renunciar a la vida laboral, los chicos consideran que se debería estudiar, y no asumir por defecto que debiera ser la madre:

"Chico: Mi madre tenía trabajo, y buenas notas y rechazó el trabajo por estar con nosotros" (Grupo 5).

"Chica: Yo preferiría que trabajara los dos, porque el trabajo a la hora de la realización profesional..., porque no tener trabajo puede ocasionar problemas tanto económicos, como personales. Una persona cuando trabaja es más estable, cuando los dos trabajan salen de casa se despejan, es más rico, se respetan, yo creo que es más sano” (Grupo 7).

Los chicos consideran que se están produciendo cambios sociales que favorecen que se produzca una mayor igualdad. La incorporación de la mujer al mundo laboral se valora como una de las causas que puede promover un mayor equilibrio: 
"Chico: ... Yo creo que la igualdad implica, es un signo de evolución, quiero decir que cuanto más avanzada está una sociedad más se reconoce. Igualdad de derechos y de obligaciones y de estatus" (Grupo 8).

\section{La hora de llegada a casa}

Se encuentran claras diferencias en cuanto a la hora fijada de llegada a casa durante las salidas, para chicos y chicas. Algunos argumentos que se dan para que las chicas vuelvan antes son: mayor debilidad de estas, posibilidad de sufrir robos, agresiones sexuales, embarazos no deseados, etc. Frente a estos argumentos, algunos consideran que las chicas no corren más riesgos que los chicos. Se produce más sobreprotección hacia las chicas: los padres van a recogerla, las recogen algún hermano, les piden que vuelvan acompañadas, etc.

\section{Violencia de género}

Los chicos consideran que la violencia de género, tanto física como psicológica, se ha hecho más visible en los últimos tiempos. Los chicos piensan que ha sido posible que se produzca un incremento de la misma dado la necesidad de búsqueda de una cierta autoridad perdida por parte de los hombres. Algunos consideran que se están produciendo cambios sociales que hacen que este tipo de comportamientos no se toleren, produciéndose una mayor denuncia de los mismos.

\section{Influencia de la familia: información de los cuestionarios}

A partir de los datos desprendidos del Cuestionario evolutivo (Jiménez y Álvarez, 2003). Los chicos y chicas destacan los siguientes patrones de conducta de los padres y madres:

- Solían responder a sus preguntas y curiosidades con sencillez. Descartan que no respondieran o que dilataran la respuesta.

- Leían y escuchaban cuentos, anécdotas, aficiones y problemas.

- Conversaban con los hijos e hijas, especialmente durante las comidas.

- Apoyaba que decidieran libremente pero consultando antes.

Algunas de las conclusiones más significativas que se desprenden del Cuestionario de género (Jiménez y Álvarez, 2003) son que:

- El estilo de educación que predomina es el democrático, exigente y respetuoso con los intereses de los chicos y chicas.

- Las familias se implican en aficiones comunes, sin establecer diferencias de género en deportes y juegos (este hecho contrasta con las opiniones dadas en los grupos de discusión, en ocasiones sí que se ponían resistencias en función del género). Por otra parte, y esto si concuerda más con los grupos de discusión, los chicos consideran que sus juguetes resultaban bastante tradicionales. 
- No suelen darse diferencias de trato entre lo chicos y chicas (argumento que se confirma en los grupos de discusión), igualmente consideran que los padres dan las mismas oportunidades formativas a chicos y chicas.

- Los chicos y chicas consideran que la división de roles en su familia es adecuada (aquí también se percibían disonancias con respecto a los grupos de discusión).

- Los chicos y chicas destacan la figura de la madre como la persona más cercana, con más fuerza y sensibilidad.

- Los chicos y chicas perciben que sus padres siempre se han sentido orgullosos de ellos.

- Los chicos y chicas perciben que sus padres se preocupan por los amigos que tienen.

\section{Conclusiones}

\section{Valoración sobre los grupos de discusión}

La elección del grupo de discusión como técnica cualitativa nos ha posibilitado acceder a las percepciones, opiniones y valoraciones que los chicos tenían sobre diferentes aspectos. Hemos elegido esta técnica frente a la entrevista principalmente por la dificultad de acceso que teníamos a la muestra, y por tanto a la limitación de tiempo real de contacto con los chicos y chicas participantes.

Las entrevistas realizadas durante los grupos de discusión eran semiestructuradas, centrando la atención en varios bloques de contenidos. Como ventaja, que ya hemos señalado, nos ha permitido acceder a focos de interés que inicialmente no teníamos previstos.

Por otro lado, la organización alcanzada después del análisis de contenido de los grupos nos permitirá, en la continuación de este trabajo de investigación (el equipo ha presentado un nuevo proyecto a la convocatorias de ayudas de I+D 2006 que ha sido aprobado), dotar de una mayor estructuración a las entrevistas y centrar nuestra atención en aquellos aspectos que han quedado parcialmente tratados.

Una de las dificultades que plantean los grupos de discusión, es poder identificar y asignar un código a cada uno de los informantes, no resultando por tanto posible obtener información sobre las percepciones y opiniones individuales de las chicas y chicos. Los grupos de discusión se convierten de esta manera en una herramienta útil para identificar perfiles generales de grupo. La realización de entrevistas individuales podría ayudarnos a vencer esta limitación. Sin embargo, complementar esta técnica con otros instrumentos y procedimientos de recogida de información, principalmente cuestionarios y tests estandarizados, permite obtener una descripción individual de los chicos y chicas participantes en las diferentes variables atendidas.

Este intento de atención a las percepciones, opiniones, valoraciones, que los participantes en la investigación, alumnos y alumnas con Beca de Excelencia en la Universidad, han mostrado durante los grupos de discusión, nos han llevado a plantearnos, aunque en muchos casos, tan solo de manera exploratoria, una diversidad de aspectos que nos ayudan a conformar una visión amplia de la situaciones personales que éstos vienen viviendo, cómo las perciben, cuáles son las influencias que han recibido, qué papel juegan en la sociedad, qué expectativas encuentran, qué cambios sociales se están dando y qué cambios se están 
reclamando. El modelo explicativo que estamos empezando a esbozar se centra en las seis dimensiones que hemos apuntado anteriormente. En la figura 1 se señalan las posibles interacciones y los diferentes elementos analizados durante el proceso de análisis de la información recogida a través de los grupos de discusión.

\section{Ámbito familiar: conclusiones y cuestiones abiertas}

Como hemos podido apreciar a lo largo de la exposición de los resultados las familias de los chicos y chicas participantes en la investigación tienen iguales expectativas hacia ambos y favorecen iguales oportunidades formativas. Se suele respetar el interés de los chicos y chicas a la hora de elegir estudios, aunque se producen orientaciones por parte de las familias: atendiendo a los propios intereses de los padres y madres, y a las salidas profesionales y económicas de la carrera elegida.

En cuanto a los roles que las familias promueven entre chicos y chicas hemos analizado dos factores, por un lado el tipo de juegos y juguetes utilizados durante la infancia, y el reparto de tareas del hogar.

Con respecto a los juegos y juguetes los chicos y chicas parecen haberse centrado principalmente en juegos "típicos" de su sexo. En los casos en los que se promovía juegos o juguetes típicos del sexo contrario se han encontrado con valoraciones contrapuestas, de aceptación o rechazo y crítica, tanto por parte de los compañeros y compañeras como por parte de sus padres y madres. En el caso de los chicos se cuestionaba principalmente debido a las interferencias que jugar a juegos de chicas puede tener en el desarrollo de su identidad masculina. A pesar de ello, los chicos parecen considerar que estos hábitos sociales van cambiando, pensando que el desarrollo de capacidades específicas está muy condicionada por el tipo de juegos y actividades que se realizan, por lo que se propone la necesidad de ampliar el rango de juegos para que todos y todas tengan las mismas posibilidades de desarrollo.

Con respecto al reparto de las tareas del hogar se sigue percibiendo una mayor responsabilidad por parte de las mujeres, aunque también se encuentran perfiles contrarios, mayor responsabilidad por parte de los hombres, o familias en las que parece darse un equilibrio. El modelado por parte de los padres y madres parece ser una de las vías de aprendizaje que los chicos y chicas reclaman.

Y por último, en cuanto al estilo educativo que caracteriza a las familias de hijos e hijas con altas capacidades parece ser que predomina el democrático, exigente y respetuoso con los intereses y elecciones personales. Los padres y madres se caracterizan durante la infancia por responder a las preguntas y dudas de sus hijos con naturalidad, por motivar la lectura y por conversar sobre temas de interés compartidos.

La cuestión de base que nos planteábamos para estudiar en relación a las familias era conocer: ¿Cómo la familia influye en el desarrollo de un alto rendimiento y en el desarrollo de unas altas capacidades?

Cara a investigaciones futuras consideramos necesarios realizar entrevistas directas con las familias, tal como hemos hecho en estudios previos. Se hace necesario identificar de manera más clara cuáles son las expectativas y cómo se transmiten, cuáles son los apoyos dados y cuáles son el tipo de interacciones que se promueven en la familia. 
Por otro lado, también sería posible analizar posible relaciones entre variables, un ejemplo de estas podrían ser:

- Relaciones entre juegos y desarrollo de capacidades personales (orientación, matemáticas, habilidades sociales e interpersonales, etc.).

- Expectativas familiares, expectativas del colegio y expectativas personales.

- Expectativas, capacidades e intereses personales.

- Expectativas y mentalidades familiares, y expectativas y mentalidades sociales.

\section{Referencias bibliográficas}

Álvarez, B. (1999). "Factores de riesgo de desadaptación social en alumnos de altas capacidades". Revista Española de Orientación y Psicopedagogía, 10 (18), 279-296.

Álvarez, B. (2004). "La familia y los hijos con altas capacidades". En C. Jiménez: Diagnóstico y atención a los alumnos con necesidades especificas: alumnos intelectualmente superdotados. Madrid: MEC/Secretaría General de Educación, 99-117.

Bruner, J. (2006). El pensamiento narrativo en las ciencias sociales. Conferencia impartida en la Facultad de Psicología de la UNED, 31 de mayo.

Bruner, J. S. (1990). Acts of meaning. Harvard: President and Fellows of Harvard College. (Trad. cast. J. C. Gómez Crespo y J. Linaza: Actos de significado. Más allá de la revolución cognitiva. Madrid: Alianza Editorial, 2000).

Gadner, H. (1983). Frames of mind: the theory of multiple intelligences. New York: Basic Books.

Gadner, H. (1999). A multiplicity of intelligences. Scientific American, 9 (4), 19-23.

Gagné, F. (1991). "Toward a differentiatied model of gitedness and talent". En D. Colangelo y G. A. Davis: Handbook of gited education. Massachusetts: Allyn and Bacon,65-80.

Guilford, J. P. (1967). The nature of intelligence. New York: MacGraw-Hill (Trad. cast.: La naturale$z a$ de la inteligencia humana. Barcelona: Paidós).

Jiménez, C. (2000). Diagnóstico y educación de los más capaces. Madrid: UNED.

Jiménez, C. (2002). La atención a la diversidad a examen: la educación de los más capaces en el sistema escolar. Bordón, Revista de Pedagogía, 54 (2-3), 219-239.

Jiménez, C. (Responsable), Álvarez, B., Gil, J. A. y Jiménez, R. (2001). Prácticas educativas y actitudes y logros del alumno desde la perspectiva del género y la alta capacidad. III Plan Nacional de Investigación Científica y Desarrollo Tecnológico. MTAS. Instituto de la Mujer. Departamento de Métodos de Investigación y Diagnóstico en Educación. Facultad de Educación. UNED. Inédito.

Jiménez, C. (Responsable), Álvarez, B., Gil, J. A., Murga, Ma A. y Téllez, J. A. (2002a). Educación, alta capacidad y género: Alumnas y alumnos con Premio Extraordinario de Bachillerato. Plan Nacional de Investigación Científica y Desarrollo Tecnológico. Programa Sectorial de Estudios de la Mujer y del Género. Instituto de la Mujer. Departamento de Métodos de Investigación y Diagnóstico en Educación. Facultad de Educación. UNED. Proyecto bianual 2002-2004 (Disponible en: http://www.mtas.es/mujer/mujeres/estud_inves/674.pdf).

Jiménez, C. (Responsable), Álvarez, B., Gil, J. A., Murga, Ma A., Téllez, J. A. y Trillo Miravalles, Ma P. (2004). Educación, capacidad y género. Alumnos y alumnas con Premio Extraordinario de Bachillerato en la Comunidad de Madrid. Convocatoria 2004 de Proyectos de Investigación en Humanidades, Ciencias Sociales y Económicas. Comunidad de Madrid. Orden 1452/2004, de 16 de abril. Referencia del Proyecto: 06/HSE/0002/2004. Departamento de Métodos de Investigación y Diagnóstico en Educación. Facultad de Educación. UNED. Proyecto bianual 2003-2004. 
Jiménez, C., Aguado, T., Álvarez, B., Gil, J. A. y Jiménez, R. (2002b). "Caracterización de los alumnos con Premio Extraordinario de Bachillerato". Bordón, Revista de Pedagogía, vol. 54 (2-3), 383-398.

Jiménez, C., Álvarez, B., Gil, J. A., Murga, M. A. y Téllez, J. A. (2005). Educación, capacidad y género. Revista de Investigación Educativa, vol. 23 (2).

Mönks, F. J. y Manson, E. (2000). "Developmental Psychology and Gitedness: Theories and Research". En K. A. Heller, F. J. Mönks, R. J. Sternberg y R. F. Subotnik (ed.): International Handbook of Gitedness and Talent. London: Pergamon Press, 141-155.

Mönks, F. J. y van Boxtel, H. W. (1988). "Los adolescentes superdotados: una perspectiva evolutiva". En J. Freeman (dir): Los niños superdotados. Aspectos psicológicos y pedagógicos. Madrid: Santillana, 306-327.

Prieto, $M^{a}$ D. y col. (Ballester, P. y Ferrándiz, C.) (2004). "Evaluación de talentos específicos desde el modelo de las inteligencias múltiples". En C. Jiménez: Diagnóstico y atención a los alumnos con necesidades especificas: alumnos intelectualmente superdotados. Madrid: MEC/Secretaría General de Educación, 45-66.

Prieto, Ma D., Ferrándiz, C. y Ballester, P. (2002). "Inteligencias múltiples y talentos específicos". Bordón, Revista de Pedagogía, vol. 54 (2-3), 283-295.

Renzulli, J. S. (1994). "Desarrollo del talento en las escuelas. Programa práctico para el total rendimiento escolar mediante el modelo de enriquecimiento escolar". En Y. Benito (coord.): Intervención e investigación psicoeducativa en alumnos superdotados. Salamanca: Amarú Ediciones, 175-217.

Renzulli, J. y Park, S. (2000). "Gifted Dropouts: the Who and the Why". Gifted Child Quaterly, vol. 44 (4), 261-271.

Seeley, K. R. (1993). "Gifted students at risk". En L. K. Silverman (ed.) Counseling the gifted and talented. Denver: Love Publishing Company, 263-275.

Spearman, C. (1927). The abbilities of man. Londres: MacMillan.

Sternberg, R. (1991). "Giftedness according to the Triarchic Theory of Human Intelligence". En D. Colangelo y G. A. Davis: Handbook of gited education. Massachusetts: Allyn and Bacon,45-54.

Tannenbaum, A. J. (1991). "The social psychology of gitedness". En D. Colangelo y G. A. Davis: Handbook of gited education. Massachusetts: Allyn and Bacon, 27-44.

Téllez, J. A. (2005). La comprensión de los textos escritos y la psicología cognitiva. Más allá del procesamiento de la información. Madrid: Dykinson.

Thustone, L. L. (1938). "Primary mental abilities". Psychometric Monograph, 1 (Trad. cast.: Aptitudes mentales primarias. Madrid: TEA, 1976).

Fecha de recepción: 28-09-05

Fecha de revisión: 12-01-07

Fecha de aceptación: 08-05-07 
